\title{
Hemorragia intracraneal secundario a déficit de factor $V$. A propósito de un caso
}

\section{Intracranial haemorrhage secondary to factor $V$ deficit - a case report}

\author{
Mariangela Velásquez', Diego A. Echenique ${ }^{1}$, Nafxiel Brito', Francisco Díaz', Edenys L. Izaguirre', \\ Luis M. Campos', Magalys Tabasca', María Lucy De Gouveia', Krivoy Jaime'
}

${ }^{1}$ Cátedra y Servicio de Neurocirugía, Hospital Universitario de Caracas. Caracas, Venezuela.

\begin{abstract}
Resumen
El déficit de factor $V$ es un trastorno hemorrágico poco común que sólo se presenta en 1/1millón de habitantes, puede aparecer en cualquier edad, manifestándose de forma más grave durante la infancia. Puede ser congénita o adquirida, y pasar inadvertida durante toda la vida o presentarse clínicamente de dos formas: hemorrágica o la variedad trombótica (factor $\mathrm{V}$ de Leyden), por mutación en sitio de escisión de la proteína $\mathrm{C}$ al factor $\mathrm{V}$. No existe hasta ahora tratamiento disponible con factor $V$, por lo que el mismo se basa en plasma fresco congelado y unidades de plaquetas. El presente caso trata de un lactante menor con sangrado en el sistema nervioso central (SNC) como único signo de la enfermedad, de presentación severa, asociada a prolongación de tiempos de coagulación, que tras múltiples complicaciones respondió satisfactoriamente al tratamiento médico-quirúrgico aplicado.
\end{abstract}

Palabras clave: Factor V, factor V de Leyden, Proteína C, Plasma fresco congelado.

\section{Abstract}

Factor $\mathrm{V}$ deficiency is a hemorrhagic disorder that occurs only in 1 case /1million habitants, it: It appears at any age, with special impact on childhood; it can be congenital or acquired, and can be asymptomatic throughout lifetime or debut in each two of both ways: hemorrhagic or the thrombotic variety (Factor $\mathrm{V}$ Leyden), by mutation at site of cleavage of protein $\mathrm{C}$ to factor $\mathrm{V}$. It does not exist so far available treatment with factor $\mathrm{V}$, so it is based on fresh frozen plasma and platelet units. The present case is a minor infant with central system nervous bleeding as the only sign of the disease, with severe presentation, associated with prolongation of coagulation times, which, after multiple complications, responded satisfactorily to the medical-surgical treatment applied.

Key words: Factor V, Factor V of Leyden, Protein C, Fresh frozen plasma.

\section{Correspondencia a:}

Nafxiel Jesús Brito-Núñez

Avenida los Ilustres. Ciudad Universitaria.

Cátedra y Servicio de Neurocirugía.

Hospital Universitario de Caracas. Venezuela.

Teléfono +584148831685

nafxiel@gmail.com 


\section{Introducción}

El déficit de factor $\mathrm{V}(\mathrm{FV})$ es un trastorno hemorrágico que se caracteriza por un amplio espectro de signos, que van desde sangrado mucocutáneo hasta compromiso del sistema nervioso central (SNC) ${ }^{1,2}$; puede ser congénita o adquirida, se caracteriza por ser una alteración autosómica recesiva, también conocida como parahemofilia; tiene diferentes presentaciones clínicas, dentro de las cuales están sangrado mucocutáneo, hematomas y hemorragia uterina anormal; así mismo se presenta de forma adquirida, siendo esta última más común y se origina debido a la producción de anticuerpos que se unen al FV, los cuales promueven su degradación o bloquean su actividad ${ }^{2}$.

Este déficit es una entidad poco frecuente con una incidencia de un caso por cada millón de habitantes ${ }^{2,3}$. Diversos estudios detectaron que esta mutación existió en $40 \%$ de los pacientes con tromboembolismo venoso, siendo los mayores de 70 años quienes presenten la mutación en comparación con los más jóvenes ${ }^{1}$, sin embargo, puede aparecer a cualquier edad, pero las formas más graves se manifiestan durante la infancia ${ }^{4}$. La presencia del FV Leiden y la mutación del gen de la protrombina (F2G20210A) son las afecciones más comunes, y es asociada con aumento del riesgo de desarrollo de trombosis venosa profunda y tromboembolismo pulmonar entre el 5 a $80 \%$, este último dato respecto al estado homocigoto en el FV Leiden (FVL). Mientras que en la deficiencia de proteína $C$, proteína $S$ y antitrombina III el riesgo llega hasta el $20 \%$, siendo la homocigosis de la proteína $\mathrm{S}$ la que peor resultado clínico presenta, por tanto, la presencia de heterocigosis $u$ homocigosis determina tanto el resultado clínico como la variabilidad de la presentación ${ }^{5}$.

El FVL es una mutación puntual del factor $V$ que resulta en una eliminación del sitio de escisión en el factor $\mathrm{V}$ y el factor $\mathrm{Va}$. Este defecto genético conlleva un mayor riesgo de trombosis, especialmente en mutaciones FVL homocigotas o pseudo-homocigotas. Muchas personas con la mutación nunca desarrollarán un evento trombótico venoso, en cambio el déficit del FV conlleva a eventos hemorrágicos ${ }^{6}$.

La forma de presentación clínica de esta enfermedad puede variar desde ser totalmente asintomático y pasar desapercibido durante toda la vida, diagnosticándose incidentalmente, o puede ser mortal con presentación de sangrado en el sistema nervioso central, principalmente de forma perinatal. La frecuencia de sangrado en el sistema nervioso central es de tan sólo $8 \%$ de los sitios de sangrado secundarios a deficiencia de factor $\mathrm{V}^{2}$, lo que hace infrecuente de observar como causa de hematomas intracraneales, esto realza la importancia del estudio de esta patología, tomando en cuenta que su desenlace puede ser mortal si no es diagnosticado y tratado a tiempo. Esto debe forzar a considerar esta etiología al momento de estudiar hematomas intracraneales espontáneos sobre todo en niños ${ }^{1-3,7}$. El objetivo del presente trabajo es comunicar un caso clínico de un lactante menor con déficit del factor $\mathrm{V}$ cuya primera manifestación fue la presentación espontánea de hematoma subdural e intraparenquimatoso simultáneos.

\section{Presentación del caso}

Paciente masculino lactante menor de 5 meses, cuya madre refiere inicio de enfermedad actual 15 días antes de la evaluación en la Cátedra y Servicio de Neurocirugía del Hospital Universitario de Caracas, caracterizado por vómitos en 8 oportunidades en 4 horas, acompañado por convulsiones tónico clónicas generalizadas en 2 oportunidades, movimientos coreicos continuos en hemicuerpo derecho, y deterioro progresivo del estado de consciencia; en los antecedentes perinatales y obstétricos, madre refiere hipertensión inducida por el embarazo controlada, fue obtenido por parto vaginal a las 38 semanas, sin complicaciones, ni otro antecedente de importancia. Examen físico se observa paciente somnoliento, con un puntaje en la escala de coma de Glasgow (adaptado a pediátricos) $)^{8}$ de $6 / 15$ puntos dados por ausencia de llanto, ninguna respuesta ocular y retira al estímulo doloroso, anisocoria con dilatación pupilar izquierda ( $5 \mathrm{~mm}$ en ojo izquierdo y $2 \mathrm{~mm}$ en ojo derecho), y poca respuesta al estímulo luminoso en el mismo, además, movimientos coreicos mantenidos en hemicuerpo derecho; no se observan signos clínicos ni radiológicos de traumatismo craneal.

Se realizan estudios de laboratorios donde se observa prolongación de tiempos de coagulación (tiempo parcial de tromboplastina incoagulable) y descenso de la hemoglobina, también se lleva a cabo Resonancia Magnética (RM) cerebral (Figura 1), donde se observa en la región frontal izquierda una imagen intraparenquimatosa de bordes irregulares, de 60 x $50 \mathrm{~mm}$ de diámetro, que comprime y colapsa asta anterior de ventrículo lateral izquierdo; en ponderación T1 y T2 se observa de intensidad heterogénea, de predominio hipointenso en T1 y predominio hiperintenso en T2; además se evidencia imagen de colección subdural subaguda tardía ${ }^{9}$ de ubicación frontoparietotemporal izquierda de $14 \mathrm{~mm}$ de espesor hiperintensa en ponderación T1 y T2, con un componente frontal hipointenso; además se observa dilatación de ventrículos laterales y tercer ventrículo, con desplazamiento de línea media de $20 \mathrm{~mm}$.

Es llevado a mesa operatoria de emergencia donde se le realiza abordaje hemicoronal izquierdo, más craneotomía frontal izquierda y durotomía frontal ipsilateral, encontrándose hematoma subdural frontoparietotemporal izquierdo representado por líquido amarillo con escasos coágulos de $40 \mathrm{cc}$ aproximadamente, además se halla hematoma intraparenquimatoso frontal ipsilateral con coágulos de 50 cc aproximadamente, se realiza drenaje de ambos hematomas con lavado de áreas de colección y realización de hemostasia (Figura 2), el flap óseo se fija con Polyglactin 910 (Vicryl) número 0.

En el postoperatorio inmediato se observa marcada mejoría del paciente con recuperación total del estado de consciencia, escala de coma de Glasgow de 15 puntos, pupilas isocóricas con buena respuesta a la luz, y persistencia de movimientos coreicos en hemicuerpo derecho para la cual se inició anticonvulsivo tipo Difenilhidantoína a $15 \mathrm{mg} / \mathrm{kg} / \mathrm{día}$, lográndose 3 días después la desaparición de los mismos.

Así mismo en el control imagenológico a las 6 semanas del postoperatorio (Figura 3), se observó la formación de higroma subdural en sitio de ubicación de hematoma subdural frontoparietotemporal y dilatación ventricular debido a estenosis del acueducto de Silvio, además comienza a presentar 


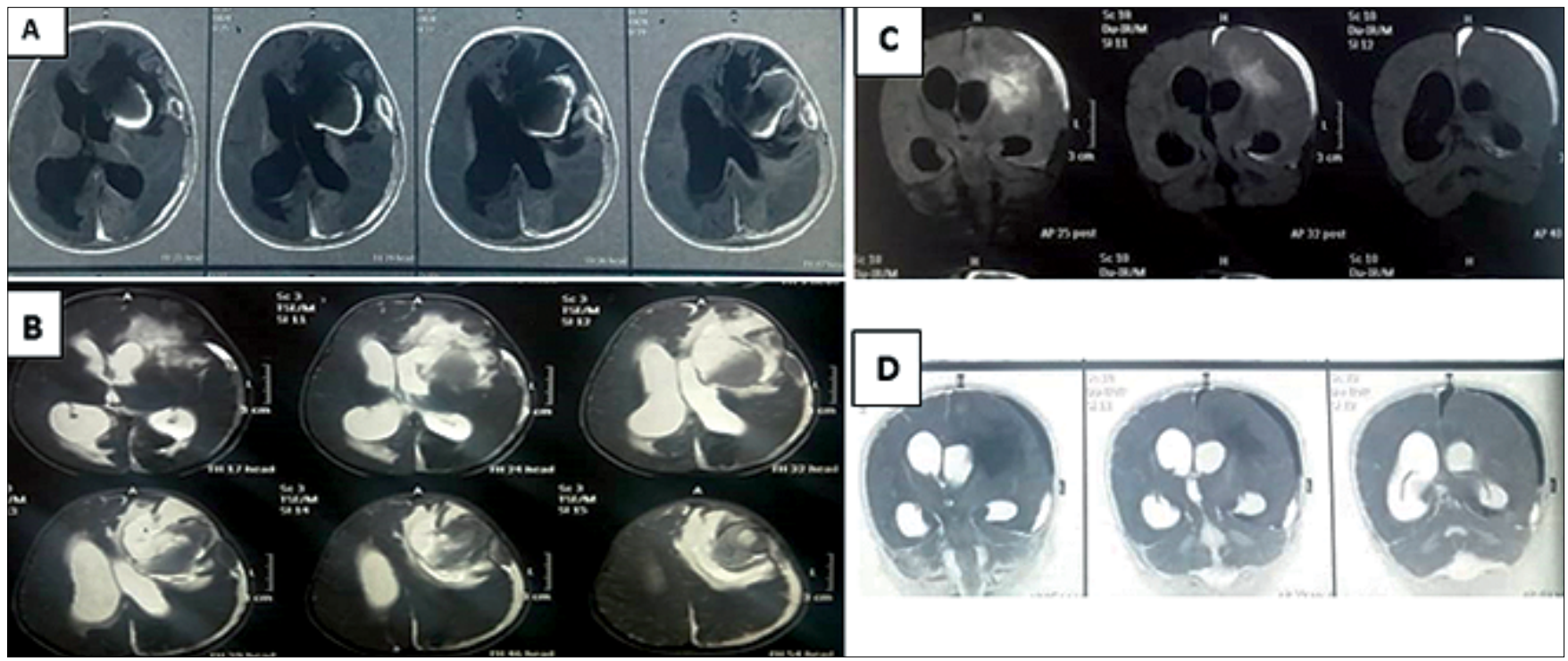

Figura 1. RM cerebral sin contraste preoperatoria. A. Ponderación T1, corte axial; B. Ponderación T2, corte axial; C. Ponderación T2 FLAIR corte coronal; D. Ponderación T2, corte coronal. Se observa hematoma intraparenquimatoso frontal izquierdo y hematoma subdural frontoparietotemporal izquierdo.

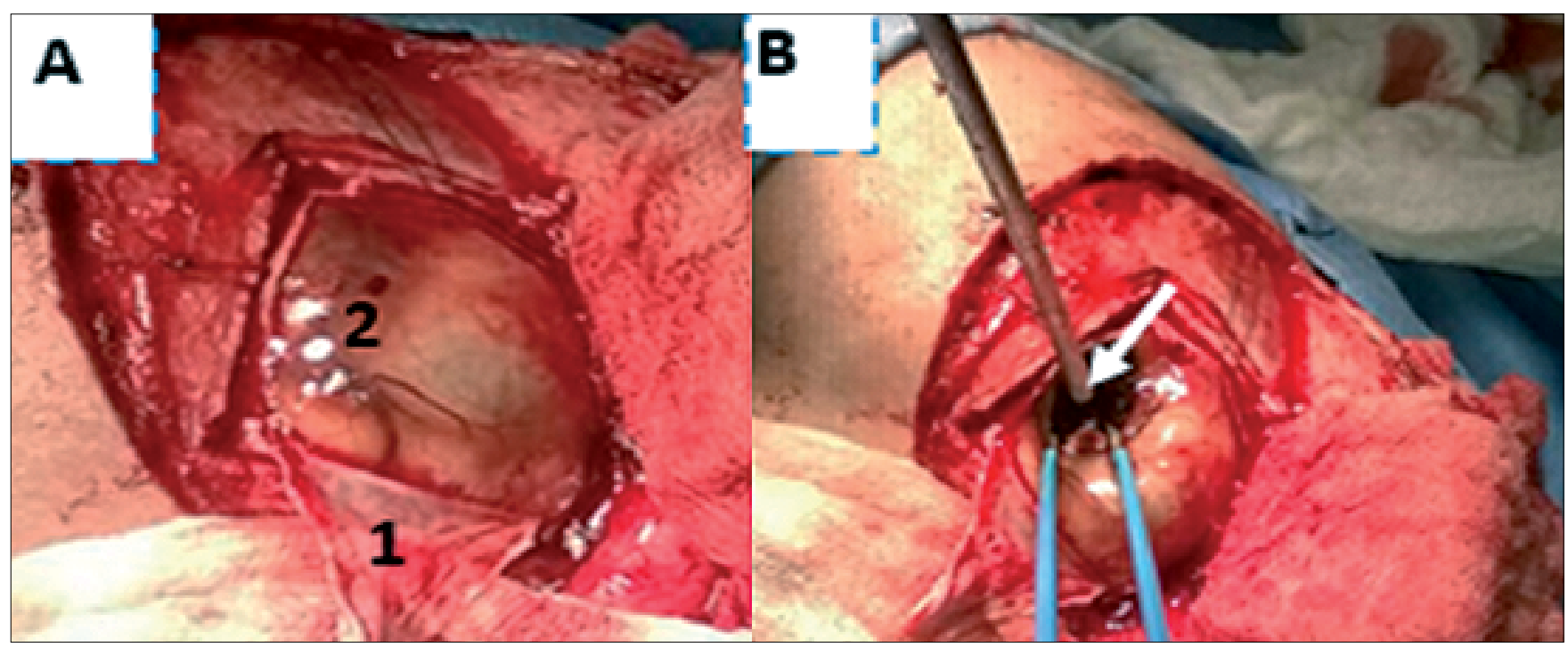

Figura 2. Imágenes transoperatorias. A. Posterior a durotomía (1) observe la membrana externa del hematoma subdural (2); B. Observe el hematoma intraparenquimatoso ya drenado (flecha).

clínica de hipertensión edocraneana dado por vómitos en proyectil, somnolencia que alterna con períodos de llanto incontrolable, y se observa levantamiento subcutáneo de flap óseo de craneotomía frontal izquierda (Figura 4), por lo que, se realiza nueva intervención quirúrgica donde se le coloca sistema de derivación ventriculoperitoneal de presión media en punto de Kocher izquierdo conectado con sistema en $Y$ con catéter subdural frontal en higroma subdural, procedimiento realizado bajo la guía de eco intraoperatorio; observándose mejoría total de la sintomatología del paciente, sin déficit postoperatorio, y en Tomografía computarizada (TC) cerebral postoperatoria inmediata (Figura $5 \mathrm{~A}-\mathrm{C}$ ), donde se observa disminución de la talla ventricular y del tamaño del higroma subdural; además, se comprueba ubicación correcta de catéter ventricular y subdural respectivamente. Se realiza también angiotomografía (angioTC) cerebral sin nuevos hallazgos de importancia.

Así mismo, en búsqueda de la etiología de los hematomas espontáneos presentados en el paciente, se realizó estudios para determinar valores de factores de coagulación y estudiar la presencia de alguna coagulopatía obteniéndose en déficit del factor $\mathrm{V}$, por lo que se inició tratamiento para este déficit a través de la administración de plasma fresco congelado a una dosis de $10 \mathrm{cc} / \mathrm{kg} /$ peso durante el pre, trans y postoperatorio del paciente y luego cada 8 horas por 7 días, así como también ácido amino-capróico $60 \mathrm{mg} / \mathrm{kg}$ cada 6 horas y ácido tranexámico $15 \mathrm{mg} / \mathrm{kg}$ cada 8 horas; además alertándose al familiar de las posibles complicaciones y recidivas de 


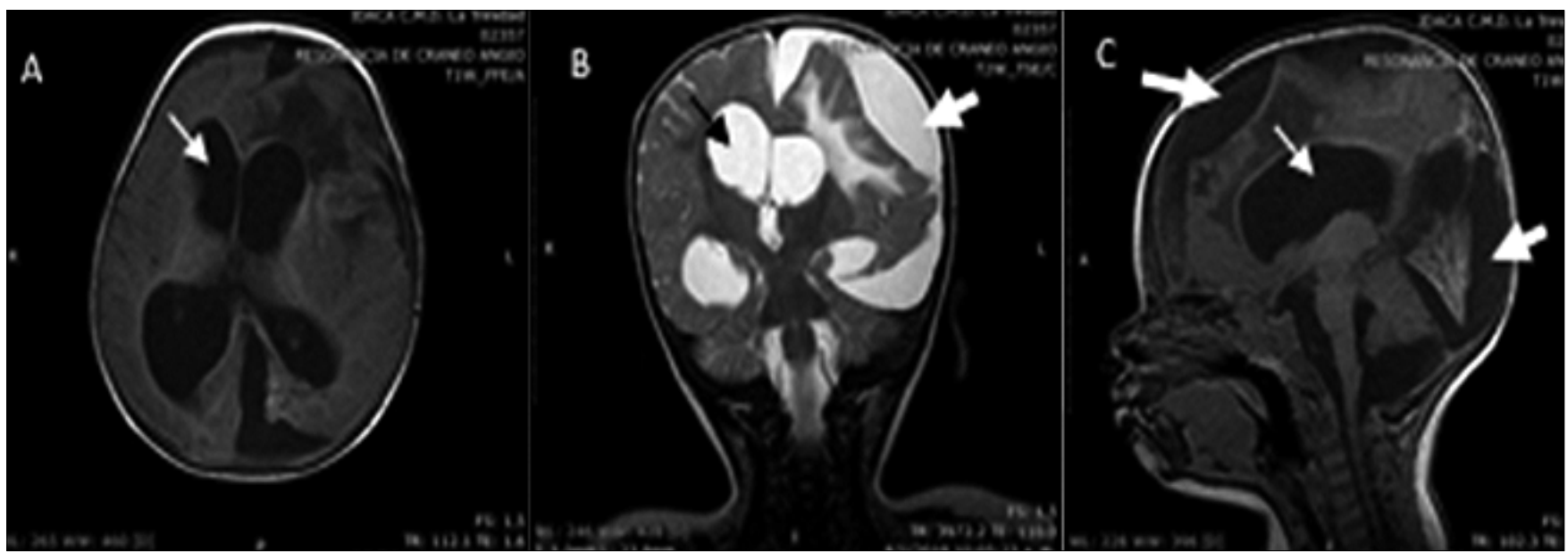

Figura 3. RM cerebral en postoperatorio tardío. A. Se observa dilatación ventricular (flecha blanca); B. Se observa el higroma subdural (flecha blanca); C. Corte sagital observe el higroma subdural en frontal y occipital (flechas blancas gruesas).

sangrado intracraneales y en otro sistema que pueden presentarse en el paciente por dicha coagulopatía y las acciones que deben tomarse para prevenirlas, se le realizó estudios de angioRM cerebral y no se encontraron malformaciones vasculares asociadas. En los controles imagenológicos al año de la colocación del sistema de derivación ventricular y subdural no se observa recidiva del sangrado, con cambios de encefalomalacia en región frontal izquierda (Figura 5 D-F). Actualmente, a los 2 años de vida el paciente se encuentra bajo manejo y control multidisciplinario con pediatría, hematología, neurología pediátrica y neurocirugía, sin déficit psicomotor ni convulsiones.

\section{Discusión}

EI FV de coagulación es una glucoproteína grande de $330 \mathrm{kD}$ sintetizada principalmente en el hígado y en los megacariocitos, su vida media es de 12 a 36 horas $^{2}$. El $80 \%$ de FV circula en el plasma, mientras que el $20 \%$ restante se almacena dentro de los gránulos $\alpha$ de las plaquetas. En su forma activa (FVa), es un cofactor esencial para el factor $\mathrm{Xa}$, que ayuda a la conversión de protrombina en trombina. La actividad procoagulante de FVa está regulada por la proteína $C$ activada (PCA), que escinde múltiples enlaces peptídicos en FVa. La escisión de FV por PCA en el residuo p.Arg534 ("Arg506" en la nomenclatura heredada) convierte el FV en una proteína anticoagulante (FVac) que carece de actividad procoagulante y sirve como un cofactor para PCA, que en sinergia con la proteína S media la degradación de FVIIla. EI FV también es portador y factor de coagulación del inhibidor de la vía del factor tisular inhibidor de la coagulación, por lo tanto, los defectos en FV pueden dar lugar a fenotipos hemorrágicos (déficit de factor $\mathrm{V}$ ) o trombóticos (factor $\mathrm{V}$ de Leyden) ${ }^{7}$.

La sospecha diagnóstica de esta entidad así como de diversos déficits congénitos de factores de coagulación, se realizará ante un neonato sano con una hemorragia inexplicable y con estudio de coagulación alterado (TP y/o TPTa

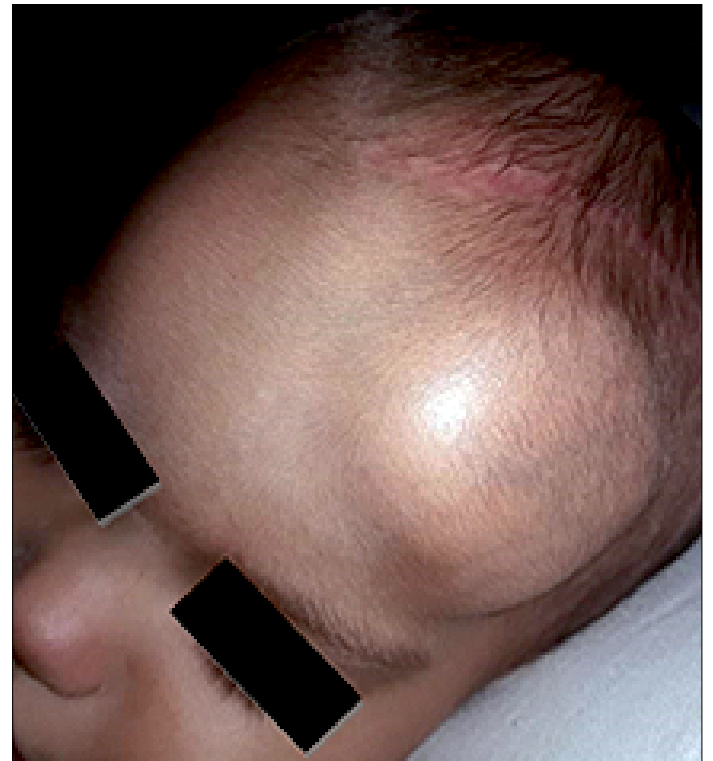

Figura 4. Levantamiento del Flap óseo frontal.

dependiendo del nivel al que actúe el factor deficiente $)^{10}$. Para detectar las mutaciones del factor V de Leiden y G20210A de la protrombina debe realizarse estudio genético al recién nacido y a los padres. En la madre además debe investigarse la presencia de anticuerpos antinucleares, anticoagulante lúpico y anticuerpos anticardiolipina ${ }^{10}$. En definitiva, en esta entidad se observa que tanto el TP como el TPT son prolongados, y el diagnóstico se confirma realizando un ensayo del FV, así también debería realizarse un ensayo del FVIII para descartar una deficiencia combinada ${ }^{3}$. El valor normal de Factor $V$ es de 71-125 Ul/dl, considerándose valor hemostático de este factor de 10-15 o de 15-20 U/dl según diversos autores, y deficiencias graves cuando tienen niveles de FV de $<1$ a $10 \mathrm{U} / \mathrm{dl}$, en las cuales tienen una tendencia hemorrágica que aparece en la infancia con propensión a moretones y hemorragias en membranas mucosas, particularmente epistaxis ${ }^{3}$. 


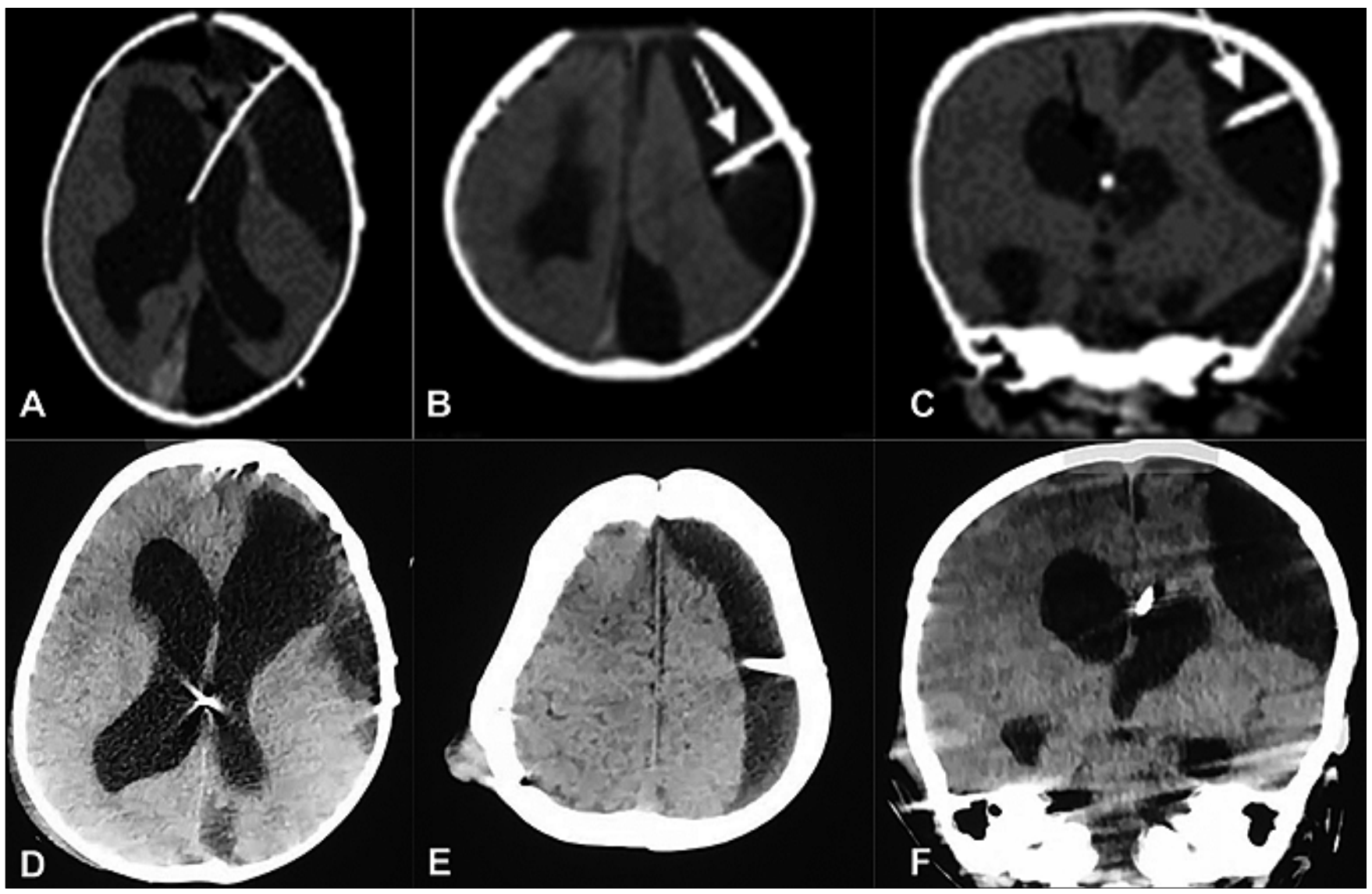

Figura 5. TC cerebral postoperatoria inmediata (A-C). Catéter ventricular (flecha negra $[A]$ ), Catéter subdural (flecha blanca $[B]$ ), de sistema de derivación ventriculoperitoneal de presión media izquierda. TC control al año postoperado (D-F), observe la encefalomalacia frontal izquierda (D) y el higroma subdural (E y F).

Durante el planteamiento diagnóstico es importante también realizar una buena anamnesis para lograr diferenciar si se trata de deficiencia de factor $V$ congénita o adquirida, por ejemplo, la deficiencia de factor $V$ adquirida, que se produce por anticuerpos o administración de trombina bovina, se manifiesta principalmente por sangrado postoperatorio, en cuyos casos se requiere la medición de títulos de anticuerpos ${ }^{2}$.

Además de todos estos exámenes de laboratorios, ante sospecha de hemorragia intracerebral con deterioro del estado neurológico del paciente o focalización neurológica es necesario realizar estudios de imagen de TC y/o RM cerebral para evaluar la presencia de hematomas intracraneales ${ }^{11}$, originados espontáneamente en este caso por la presencia de déficit de factor $\mathrm{V}$. Escudero et al. ${ }^{12}$; afirman que, aunque tanto la TC como la RM craneal pueden utilizarse en el diagnóstico de hematoma intraparenquimatoso para determinar su tamaño, localización y crecimiento, la TC sigue siendo la técnica de elección, ya que presenta una sensibilidad y especificidad cercanas al 100\%; además permite documentar la evolución del sangrado ${ }^{11,12}$. Es necesario así mismo durante el estudio de hematomas intracraneales, en particular en edades pediátricas, realizar diagnóstico diferencial con respecto a posibles causas del hematoma espontáneo como malformaciones arteriovenosas (MAV), para lo cual están disponibles los estudios de neuroimágenes (angioTC cerebral, angioRM cerebral y la arteriografía cerebral con substracción digital), de las cuales la angioRM proporciona mayor información, debido a que es capaz de detectar lesiones que son angiográficamente ocultas y tener precisión de la edad evolutiva del hematoma ${ }^{9,11,12}$.

Así mismo, en vista de que se ha informado de neonatos con hematomas intracraneales (HIC), es prudente vigilar atentamente a bebés y realizar un ultrasonido craneal durante los primeros días de vida ${ }^{3,10}$, como parte de control y prevención de hemorragias intracraneales asociadas a recién nacidos con déficit de factor $\mathrm{V}$, el cual además puede realizarse como seguimiento ante la presencia de hematoma intracraneal detectado en un estudio previo, en casos en que la TC cerebral sea de difícil acceso ${ }^{10}$.

El tratamiento del déficit de factor $V$ se basa en la administración de plasma fresco congelado y de unidades de plaquetas, sin embargo, las plaquetas solo se utilizan en casos de deficiencia clasificada como severa o en pacientes con inhibidores de factor $\mathrm{V}^{2}$. El tratamiento disponible y preferido hasta la actualidad para este trastorno es el Plasma Fresco Congelado (PFC), de preferencia inactivado viralmente, ya que no hay concentrados que contengan $\mathrm{FV}^{3}$. Es de gran importancia conocer que el nivel hemostático mínimo es de $15 \mathrm{UI} / \mathrm{dl}^{3}$, y pueden necesitarse grandes cantidades de plasma para lograr este nivel, siendo la dosis PFC de 10-20 $\mathrm{ml} / \mathrm{kg}$ de peso ${ }^{1}$. La transfusión de plaquetas (con FV en los gránulos) puede ser provechosa ${ }^{7}$ en una dosis de $1 \mathrm{Ul} / 10 \mathrm{~kg}$ 
de peso'. Aquellos con niveles de factor $\mathrm{V}<1 \%$, o quienes van a ser llevados a un procedimiento quirúrgico se debe garantizar una actividad de factor $\mathrm{V}$ aproximadamente del $20 \%^{2}$. La vida media de dicho factor es de 12 a 36 horas y esta meta se logra con administración diária o cada 12 horas de plasma fresco congelado $15-20 \mathrm{ml} / \mathrm{kg}$. En pacientes que presentan además hemorragia uterina anormal, se recomienda la utilización de ácido amino-capróico (50-60 mg/kg cada 4 a 6 horas), ácido tranexámico ( $15 \mathrm{mg} / \mathrm{kg}$ cada 6 a 8 horas), o terapia hormonal tipo anticonceptivos orales o levonorgestrel mediante dispositivos intrauterinos ${ }^{2}$.

El pronóstico en pacientes que padecen este trastorno generalmente es bueno, cuando el diagnóstico es temprano y el tratamiento adecuado 4 , sin embargo, en ocasiones puede ser mortal, cuando se presenta con sangrado en el sistema nervioso central, principalmente de forma perinatal ${ }^{2}$. Por lo tanto, al momento de la sospecha diagnóstica de esta patología deben tomarse las medidas de tratamiento oportunas y adecuadas, así como los cuidados preventivos de sangrado en el paciente, sobre todo si va a ser sometido a alguna intervención quirúrgica, y si ya ha presentado el sangrado en SNC aplicar las medidas correctivas necesarias para preservar la vida del paciente.

En González et al. 2017,13 presentaron un caso de una paciente con déficit del factor $V$ que fue tratada de forma regular con trasfusiones de PFC, que a los 7 años presentó caída de sus pies y desarrolló un hematoma subdural de 22 $\mathrm{mm}$ de espesor, sin embargo, la paciente no hizo deterioro neurológico y fue manejado medicamente con las transfusiones de PFC con buena respuesta clínica, a diferencia del presenta caso que presentó deterioro neurológico que justificó el tratamiento quirúrgico.

En el presente caso a pesar de las estadísticas apuntar a mal pronóstico, este paciente respondió de forma sorprendente, evolucionando además de forma adecuada ante cirugías posteriores para colocación de sistema de derivación ventrículo peritoneal para tratar la hidrocefalia y el higroma subdural subsecuente al drenaje del hematoma subdural, tomando las previsiones establecidas para mantener niveles hemostáticos de factor $\mathrm{V}$ adecuados ante una cirugía con la ayuda de transfusión de PFC. El diagnóstico se comprobó con estudio de valores de factor $V$ disminuidos. Así mismo se demuestra en este caso que este trastorno puede presentarse de una forma muy compleja, con características clínicas inusuales y respuesta muy satisfactoria ante el tratamiento aplicado a pesar de ser de presentación severa, si el mismo es instaurado de forma oportuna y correcta.

\section{Referencias}

1. Robles L, Pera R, Boladeras B. Trastornos de la coagulación. In: Trastornos de La Coagulación. Tratado de Geriatria Para Residentes. Situaciones Clínicas Más Relevantes. Madrid: Sociedad Española de Geriatría y Gerontología; 2017:679687.

2. Aguirre H, Posada A, Aponte J. Deficiencia congénita de factor V. Rev CES Med. 2014;28(2):247-252. http://www.scielo.org.co/ pdf/cesm/v28n2/v28n2a09.pdf.

3. Peyvandi F, Bolton-Maggs $\mathrm{PH}$, Batorova A, De Moerloose P. Rare bleeding disorders. Haemophilia. 2012;18:148-153. doi:10.1111/j.1365-2516.2012.02841.x

4. FMH. ¿Qué son las defi ciencias poco comunes de factores de la coagulación? http://www1.wfh.org/publication/files/pdf-1338. pdf. Published 2009. Accessed May 1, 2019.

5. Noroña C. Trombofilias hereditarias. Rev Cient Cienc Med. 2015;18(1):43-49. http://www.scielo.org.bo/pdf/rccm/v18n1/ v18n1_a09.pdf.

6. Albagoush SA, Schmidt AE. Factor V Leiden Deficiency.; 2019. http://www.ncbi.nlm.nih.gov/pubmed/30521223.

7. Sridharan M, Coon L, Chen D, Pruthi R. Factor V Deficiency with a Thrombotic Clinical Phenotype. Semin Thromb Hemost. 2019;45(01):108-112. doi:10.1055/s-0038-1677041

8. Silva Higuero N, García Ruano A. Traumatismos craneoencefálicos. Pediatr Integr. 2014;XVIII(4):207-218. https://www. pediatriaintegral.es/wp-content/uploads/2014/xviii04/01/207-218. pdf.

9. Gálvez M, Bravo E, Rodríguez P, Farías M, Cerda J. Caracteísticas de las hemorragias intracraneanas espontáneas en TC y RM. Rev chil radiol. 2007;13(1):12-25. doi:10.4067/S071793082007000100005

10. Guzmán J, Gómez E, Martínez MD, Ruiz MD, Parraga MJ. Trastornos de la coagulación en el recién nacido. Asoc Española Pediatría. 2008. https://www.aeped.es/sites/default/files/ documentos/40.pdf.

11. Rodríguez-Yáñez M, Castellanos M, Freijo MM, et al. Guías de actuación clínica en la hemorragia intracerebral. Neurología. 2013;28(4):236-249. doi:10.1016/j.nrl.2011.03.010

12. Escudero AD, Marqués Álvarez L, Taboada Costa F. Actualización en hemorragia cerebral espontánea. Med Intensiva. 2008;32(6):282-295. doi:10.1016/S02105691(08)70956-2

13. González-Villén R, -leguezuelos IP, González-Espín Al, VílchezPérez JS, De la Cruz-Moreno J. Déficit de factor $V$ grave. Rev Esp Pediatr. 2017;73(2):75-78. http://www.seinap.es/wp-content/ uploads/2015/05/REP-73-2.pdf. 